\title{
BMJ Open Survival of patients with alcohol use disorders discharged from an emergency department: a population- based cohort study
}

\author{
Anna Steinunn Gunnarsdottir, ${ }^{1}$ Adalbjorg Kristbjornsdottir, ${ }^{1}$ \\ Ragnhildur Gudmundsdottir, ${ }^{1}$ Oddny Sigurborg Gunnarsdottir, ${ }^{2}$ \\ Vilhjalmur Rafnsson ${ }^{3}$
}

To cite: Gunnarsdottir AS, Kristbjornsdottir A, Gudmundsdottir R, et al. Survival of patients with alcohol use disorders discharged from an emergency department: a population-based cohort study. BMJ Open 2014;4: e006327. doi:10.1136/ bmjopen-2014-006327

- Prepublication history for this paper is available online. To view these files please visit the journal online (http://dx.doi.org/10.1136/ bmjopen-2014-006327).

Received 11 August 2014 Revised 3 November 2014 Accepted 19 November 2014

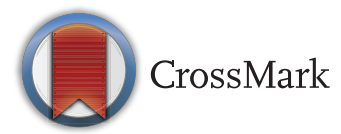

${ }^{1}$ Faculty of Medicine, The Centre of Public Health Sciences, University of Iceland, Reykjavik, Iceland ${ }^{2}$ Landspitali-the National University Hospital of Iceland, Reykjavik, Iceland ${ }^{3}$ Department of Preventive Medicine, Faculty of Medicine, University of Iceland, Reykjavik, Iceland

Correspondence to Dr Vilhjalmur Rafnsson; vilraf@hi.is

\section{ABSTRACT}

Objectives: The aim was to study the cause-specific mortality of users of the emergency department (ED) who received a diagnosis of alcohol use disorder (AUD) in comparison with mortality of other users of the department.

Design: A population-based prospective cohort study. Participants: All patients aged 18 years and above who were subsequently discharged home from the ED during the years 2002-2008. A total of 107237 patients were followed by record linkage to a nationwide cause-of-death registry: 1210 patients with AUD as the main discharge diagnosis and 106027 patients in the comparison group. HR and $95 \% \mathrm{Cls}$ were calculated.

Setting: ED at Landspitali-the National University Hospital of Iceland, Reykjavik, Iceland. The hospital offers tertiary care and is the number one trauma centre and community hospital for the greater Reykjavik area. According to the population registry, $78 \%$ of the inhabitants of the area attended the ED during the study period.

Results: 72 patients died in the AUD group and 4807 in the comparison group. The adjusted HR for all causes of death was 1.91 (95\% Cl 1.51 to 2.42). The HR for AUDs was 47.68 (95\% Cl 11.56 to 196.59) while for alcohol liver disease the HR was $19.06(95 \%$ $\mathrm{Cl} 6.07$ to 59.87). The HR was also elevated for diseases of the circulatory system: HR $2.52(95 \% \mathrm{Cl}$ 1.73 to 3.68$)$; accidental poisoning: $\mathrm{HR}=13.64,(95 \%$ $\mathrm{Cl} 3.98$ to 46.73$)$; suicide: $\mathrm{HR}=2.72(95 \% \mathrm{Cl} 1.08$ to 6.83); and event of undetermined intent: $H R=10.89$ (95\% Cl 4.53 to 26.16).

Conclusions: AUD as the discharge diagnosis at the $E D$, among patients who were not admitted to a hospital ward but discharged home, predicts increased mortality. As the results conclusively show the vulnerability of these patients, one can question whether their needs are adequately met at the ED.

\section{INTRODUCTION}

Alcohol use disorders (AUD) have been associated with excessive all-cause mortality in

\section{Strengths and limitations of this study}

A population-based prospective cohort study was conducted in a setting with uniform financing of healthcare.

- The universal use of personal identifier at the emergency department (ED) and in the nationwide mortality registry enabled record linkage, and accurate counting the number of visits to the ED and ascertainment of vital status.

- The discharge diagnosis at the ED and the cause of death (from the death certificate in the mortality registry) were systematically reported according to the International Classification of Diseases, tenth version.

- The study material is from a single healthcare institution, which limits the generalisability of the results; however, there was no competition from the same kind of institutions in the catchment area.

- The patients in the ED may have had other diagnoses in the paper records besides the main diagnosis; these were not available for this study. However, the main diagnosis at discharge, chosen by the attending physician, reflects the most important clinical conclusion of the patient's visit.

three meta-analyses ${ }^{1-3}$ and this has further been corroborated in recent populationbased cohort studies. ${ }^{4}$ Important causes of death are injuries and suicide; these are highlighted in patients presenting with AUDs. ${ }^{2} 36$ Mental disorders, other than AUDs, are associated with increased all-cause mortality ${ }^{1}{ }^{3}$ and suicide. ${ }^{367}$ Whereas alcohol dependence is significantly associated with all types of substance and psychiatric disorders, ${ }^{8}$ the co-occurrence of mental disorders and AUDs should be taken into account when studying outcomes in patients with these disorders.

Alcohol-related problems or AUDs have been described as a substantial burden on emergency departments (EDs.), ${ }^{9-12}$ and the 
increase in alcohol-related visits and suboptimal management at the ED calls for action. ${ }^{12}$ The imminent and long-term mortality risks of drunken patients in the ED have only rarely been studied, ${ }^{13}$ although mortality investigations have been undertaken repeatedly on patients with a clinical diagnosis of AUDs or persons with AUDs identified in general population surveys. ${ }^{1-5}$ Increased allcause mortality of AUD patients from the ED was found in a small study ${ }^{13}$ with excess death from injuries and suicide. The setting at the ED of Landspitali-the National University Hospital of Iceland (LUH), which is the only acute care hospital serving the population of the capital area of Reykjavik, offers a unique opportunity to study the survival of those with alcohol diagnosis through the nationwide death registry.

The aim was to study the cause-specific mortality of users of the ED-those who receive a diagnosis of AUDs in comparison with the mortality of all other users of the department-while taking into consideration other psychiatric diagnoses and frequency of visits.

\section{METHODS}

This was a prospective cohort study of all individuals who attended the ED at the LUH, and who were subsequently discharged home during the study period 20022008. The data consisted of computer records of patients aged 18 years and above, and comprised 107237 individuals and 257955 visits to the ED (an average of 2.4 visits per individual). The records contained routinely collected data on every visit, including the unique registration number of each visit, personal identification number of the patient, gender, date of birth, time and date of admission, discharge diagnosis recorded according to the International Classification of Diseases tenth revision (ICD-10), and the time and date of discharge. The LUH, a university hospital at the forefront of specialised care in Iceland, offers tertiary care and is the principal trauma centre for the whole country. The LUH and the ED were the only acute care hospital and ED operated for adults in the larger capital area of Reykjavik (the municipalities of Reykjavik, Kopavogur, Seltjarnarnes, Gardabaer, Hafnarfjordur, Alftanes and Mosfellsbaer), and for that catchment area it served as a community hospital. At the LUH, other specialised EDs for psychiatry, paediatrics as well as gynaecology and obstetrics were operated. In 2005, the midyear population aged 18 years and above in the Reykjavik capital area was $137124 .{ }^{14}$ The 107237 patients attending the ED during the study period comprised $78 \%$ of the area's inhabitants; thus, the cohort may be considered to be population-based.

Personal identification numbers were used in the record linkage of the study cohort with the National Cause-of-Death Registry to obtain information on vital status and, where applicable, the date and the cause of death according to the death certificates. The causes of death were classified according to the ICD-10.
The users of the ED had, on average, visited the department more than twice during the study period and received each time one main diagnosis that was recorded in the computer system. The diagnoses were assigned by a senior physician, or by a junior physician under the auspices of a senior, and the attending physician selected one main diagnosis as the discharge diagnosis to be entered into the computer records. All these routinely collected clinical diagnoses were according to ICD-10 criteria. Any patient who had ever received AUD diagnosis (ICD-10 codes F10) as the main diagnosis during the study period was included in the exposed group $(n=1210)$, while the 106027 patients who never received an AUD diagnosis constituted the comparison group. The total number of visits was counted per individual. The first received diagnosis was used to define the exposed group and the comparison group. The other diagnoses were also taken into account as covariates.

The follow-up started on the day that the patient was first discharged from the ED with an AUD diagnosis or in the comparison group, and ended on the date of death of the patient or on the closing date of the study, 31 December 2008, whichever occurred first.

The Cox proportional model was used to estimate HR and $95 \%$ CIs for all deaths and selected causes of death, ${ }^{15}$ comparing those in the AUD group with those in the comparison group. Person time at risk was calculated for each individual and covariates were gender, age, number of visits, year of entrance and selected discharge diagnoses. Several calculations were performedcrude comparison without any adjustment; comparison with adjustment for gender and age only; adjustment for gender, age (date of birth), number of visits and year of entrance; and several different discharge diagnoses. Gender and discharge diagnoses were introduced into the model as categorical variables and age, number of visits and year of entrance as continuous variables. After introducing age, gender, number of visits and year of entrance into the model, all these covariates were statistically significant. According to previous studies, mental disorders and use of alcohol are related, and these, in turn, are associated with mortality. Thus, mental disorders according to main diagnoses were adjusted for in the model, ${ }^{1298}$ and were statistically significant. Several other main diagnoses, including those with the highest proportion in the AUD group, were also tested as covariates in the model, such as diseases of the circulatory system, diseases of the musculoskeletal system, symptoms, signs, abnormal clinical and laboratory findings, and injuries. However, these covariates did not change the results, had less than $10 \%$ effect on the HR, and were therefore omitted from the model. We decided to show the model when adjusted for age, gender, number of visits, year of entrance and mental disorders. The subcategories of causes of death were selected in the results table when three or more deaths had occurred, and thus we displayed approximately $90 \%$ of the causes of death. Survival function for all causes of death at the 
mean of covariates (adjusted for gender, age, number of visits, year of entrance, and mental and behavioural disorders at discharge) was calculated for those in the AUD group and for those in the comparison group, and displayed as two separate curves.

\section{RESULTS}

The number of individuals aged 18-106 years attending the ED was 107237 and all together the person-years totalled 408194.

Table 1 shows the characteristics of the cohort attending the ED in the years 2002-2008. The cohort comprised 57181 men, and 50056 women. Those in the AUD group differed from those in the comparison group with regard to age and gender. In the AUD group, there were $63.7 \%$ men and $36.3 \%$ women, while in comparison group comprised $53.2 \%$ men and $46.8 \%$ women. The mean age for the AUD group was 41.5 years while it was 43.5 years for the comparison group.
Selected discharge diagnoses (other than AUD at the ED) are shown in table 2. The AUD group had a higher percentage in all the selected discharged diagnoses.

Figure 1 shows the survival function adjusted for age, gender, number of visits, year of entrance and mental or behavioural disorders at discharge from the ED among those in the AUD group (dashed line) and the comparison group (black line) for all causes of death. A greater proportion of those in the AUD group died during the study period compared with those in the comparison group.

The HR and 95\% CI of all causes of death and selected causes of death are shown in table 3, for both genders combined. The HR for all causes of death was 1.91 (95\% CI 1.51 to 2.42), for mental and behavioural disorders 7.62 (95\% CI 2.76 to 21.07); for mental and behavioural disorders due to alcohol 47.68 (95\% CI 11.56 to 196.59$)$; for diseases of the circulatory system 2.52 (95\% CI 1.73 to 3.68); for diseases of the digestive system 4.58 (95\% CI 1.95 to 10.81$)$; for chronic liver

Table 1 Baseline characteristics at the emergency department 2002-2008 among the AUD group (mental and behavioural disorders (MBD), F10) and the comparison group

\begin{tabular}{|c|c|c|}
\hline & $\begin{array}{l}\text { AUD group } \\
\mathrm{N}(\%)\end{array}$ & $\begin{array}{l}\text { Comparison group } \\
\mathrm{N}(\%)\end{array}$ \\
\hline MBD due to use of alcohol (F10) & $1210(100)$ & $106027(100)$ \\
\hline Acute intoxication due to alcohol (F10.0) & $741(61.2)$ & \\
\hline Harmful use of alcohol (F10.1) & $121(10.0)$ & \\
\hline Dependence syndrome (F10.2) & $126(10.4)$ & \\
\hline Withdrawal state (F10.3) & $97(9.2)$ & \\
\hline Withdrawal state with delirium (F10.4) & $10(0.8)$ & \\
\hline Psychotic disorder due to alcohol (F10.5) & $9(0.7)$ & \\
\hline Alcoholic dementia (F10.7) & $1(0.1)$ & \\
\hline Other MBD due to use of alcohol (F10.8) & $16(1.3)$ & \\
\hline Unspecified MBD due to use of alcohol (F10.9) & $89(7.4)$ & \\
\hline \multicolumn{3}{|l|}{ Gender } \\
\hline Men & $771(63.7)$ & $56410(53.2)$ \\
\hline Women & 439 (36.3) & $49617(46.8)$ \\
\hline \multicolumn{3}{|l|}{ Age, year } \\
\hline Mean $\pm S D$ & $41.5 \pm 17.1$ & $43.5 \pm 19.1$ \\
\hline Median, IQR (0.25; 0.75) & $41(25 ; 54)$ & $41(27 ; 57)$ \\
\hline \multicolumn{3}{|l|}{ Number of visits } \\
\hline 1 & $157(13.0)$ & 50044 (47.2) \\
\hline 2 & $199(16.4)$ & 24714 (23.3) \\
\hline 3 & $136(11.2)$ & 13057 (12.3) \\
\hline 4 & $133(11.0)$ & $7074(6.7)$ \\
\hline 5 & $89(7.4)$ & 4060 (3.8) \\
\hline 6 & $75(6.2)$ & $2350(2.2)$ \\
\hline $7+$ & $421(34.8)$ & $4728(4.5)$ \\
\hline \multicolumn{3}{|l|}{ Year of attendance } \\
\hline 2002 & 442 (36.5) & 19537 (18.4) \\
\hline 2003 & $269(22.2)$ & 20074 (18.9) \\
\hline 2004 & 166 (13.7) & $16272(15.3)$ \\
\hline 2005 & 127 (10.5) & $14248(13.4)$ \\
\hline 2006 & $92(7.6)$ & 12837 (12.1) \\
\hline 2007 & $69(5.7)$ & 11996 (11.3) \\
\hline 2008 & $45(3.7)$ & 11063 (10.4) \\
\hline
\end{tabular}


Table 2 Selected diagnosis at discharge from the emergency department after the first visit among the AUD group and the comparison group

\begin{tabular}{lcc}
\hline & AUD group & Comparison group \\
Discharged diagnosis (ICD-10) & N (\%) & N (\%) \\
\hline Mental, behavioural and neurodevelopmental disorders (F00-F99) & $153(12.6)$ & $1152(1.1)$ \\
$\quad$ Mental and behavioural disorders due to psychoactive substance use (F11-F19) & $67(5.5)$ & $247(0.2)$ \\
Diseases of the circulatory system (I00-I99) & $135(11.2)$ & $4654(4.4)$ \\
Ischaemic heart diseases (I20-I24) & $35(2.9)$ & $639(0.6)$ \\
Diseases of the musculoskeletal system (M00-M99) & $273(22.6)$ & $9010(8.5)$ \\
Symptoms, signs, abnormal clinical and laboratory findings (R00-R99) & $441(36.4)$ & $13274(12.5)$ \\
Injury of certain body regions (S00-S99) & $457(37.8)$ & $20584(19.4)$ \\
Injuries of head (S00-S02) & $111(9.2)$ & $2727(2.6)$ \\
Fracture of rib (S22.3) & $27(2.2)$ & $583(0.5)$ \\
Injuries involving multiple body regions (T00-T98) & $65(5.4)$ & $2777(2.6)$ \\
Poisoning by drugs, medicaments and biological substances (T36-T50) & $12(1.0)$ & $129(0.1)$ \\
Factors influencing health status and contact with health services (Z00-Z99) & $25(2.1)$ & $1324(1.2)$ \\
No discharge diagnoses are displayed & $89(7.4)$ & $7028(6.6)$ \\
\hline AUD, acol
\end{tabular}

AUD, alcohol use disorders; ICD-10, International Classification of Diseases tenth revision.

disease 14.69 (95\% CI 4.99 to 43.28 ); and for an alcoholic liver disease 19.06 (95\% CI 6.07 to 59.87; for external causes of injury and poisoning 4.02 (95\% CI 2.48 to 6.53); for accidental poisoning 13.64 (95\% CI 3.98 to 46.73; for suicide and intentional self-harm 2.72 (95\% CI 1.08 to 6.83); for events of undetermined intent 10.89 (95\% CI 4.53 to 26.16). The HR for all causes of death was significantly associated with gender, age, year of entry, mental diseases and frequency of visits to the ED. The HR was 1.55 (95 CI 1.23 to 1.96) when adjusted for age and gender only.

Table 4 shows the number of deaths, HR and $95 \%$ CI for all causes of death and selected causes of death among men and women separately. In the AUD group,

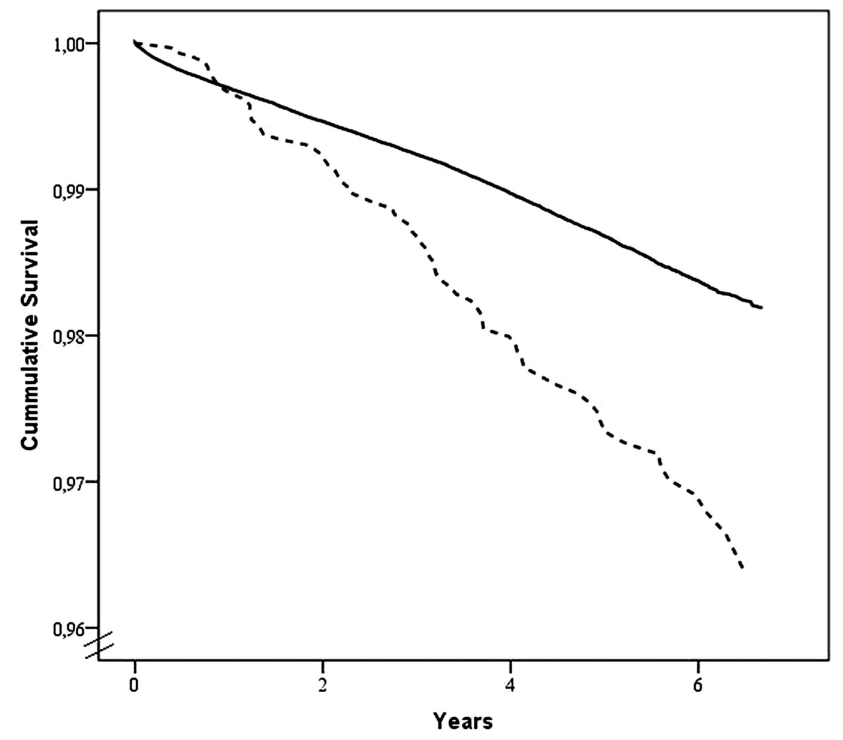

Figure 1 Survival function at mean of covariates, adjusted for gender, age, number of visits, year of entrance and mental and behavioural disorders at discharge. Dashed line indicates the alcohol use disorder group, and black line the comparison group, $p<0.0001$.
57 men and 15 women had died. The pattern of results among men was similar to the results where genders were analysed in combination, but among women, the number of deaths was too few to make any meaningful calculation for many of the causes of deaths in the model. However, these numbers are shown here for the completion.

In order to test whether patients with other alcoholrelated main diagnoses than those in F10 biased our HR, we counted the main diagnosis of alcoholic liver disease (ICD code K70) and toxic effect of alcohol (ICD-10, code T51) in the exposed and non-exposed groups. In all, 62 patients were found with these diagnoses (all in the comparison group), of whom five had died. The cause of death of each of the three patients was ICD-10, codes F10, I25, and X45, respectively, and the cause of death of the other two patients was K70. We subtracted these patients from the data and recalculated the HR corresponding to the results in table 3. The HR for all causes of death (A00-Y98) was 1.88 (95\% CI 1.48 to 2.39). For mental and behavioural disorders (F00-F99) $\quad(\mathrm{HR}=6.19$ (95\% CI 1.94 to 19.79)); for AUDs (F10) (HR=56.22 (95\% CI 12.79 to 247.13$)$ ); for diseases of the circulatory system (I00-I99) (HR=2.54 (95\% CI 1.74 to 3.72)); for ischaemic heart diseases (I20-I25) $(\mathrm{HR}=1.42(95 \% \mathrm{CI}$ 0.45 to 4.43$)$ ); for diseases of the respiratory system (J00-J99) $(\mathrm{HR}=2.12(95 \%$ CI 0.78 to 5.73$))$; for diseases of the digestive system (K00-K93) (HR=4.77 (95\% CI 2.02 to 11.24)); for chronic liver disease (K70, $\mathrm{K} 73-\mathrm{K} 74) \quad(\mathrm{HR}=16.27$ (95\% CI 5.46 to 48.46$)$ ); for alcoholic liver disease (K70) (HR=21.83 (95\% CI 6.80 to $70.08)$ ); for external causes of injury and poisoning (V01-Y98) $\quad(\mathrm{HR}=3.87$ (95\% CI 2.37 to 6.33$))$; for accidental poisoning (X40-X49) $(\mathrm{HR}=9.44$ (95\% CI 2.34 to $38.05))$; for suicide and intentional self-harm (X60-X84) $(\mathrm{HR}=2.78$ (95\% CI 1.11 to 6.95$))$; and for events of undetermined intent (Y10-Y34) $(\mathrm{HR}=11.01$ (95\% CI 4.60 to 26.33$)$ ). 
Table 3 Number of all causes and selected causes of death among the AUD group, and the comparison group, HR, 95\% Cls adjusted for age, gender, number of visits, year of entrance and mental and behavioural disorders at discharge

\begin{tabular}{|c|c|c|c|c|}
\hline Causes of death (ICD-10) & $\begin{array}{l}\text { AUD group } \\
\text { p-yr } 5703 \\
\text { N (\%) }\end{array}$ & $\begin{array}{l}\text { Comparison group } \\
\text { p-yr } 402491 \\
\text { N (\%) }\end{array}$ & HR & $95 \% \mathrm{Cl}$ \\
\hline All causes (A00-Y98) & $72(6.0)$ & 4807 (4.5) & 1.91 & 1.51 to 2.42 \\
\hline Mental and behavioural disorders (F00-F99) & $4(0.3)$ & $127(0.1)$ & 7.62 & 2.76 to 21.07 \\
\hline Alcohol use disorders (F10) & $4(0.3)$ & $6(0.0)$ & 47.68 & 11.56 to 196.59 \\
\hline Diseases of the circulatory system (I00-199) & $28(2.3)$ & $1646(1.6)$ & 2.52 & 1.73 to 3.68 \\
\hline Ischaemic heart diseases (I20-I25) & $3(0.2)$ & $366(0.3)$ & 1.41 & 0.45 to 4.41 \\
\hline Diseases of the respiratory system (J00-J99) & $4(0.3)$ & $334(0.3)$ & 2.11 & 0.78 to 5.70 \\
\hline Diseases of the digestive system (K00-K93) & $6(0.5)$ & $138(0.1)$ & 4.58 & 1.95 to 10.81 \\
\hline Chronic liver disease (K70, K73-K74) & $5(0.4)$ & $24(0.0)$ & 14.69 & 4.99 to 43.28 \\
\hline Alcoholic liver disease (K70) & $5(0.4)$ & $16(0.0)$ & 19.06 & 6.07 to 59.87 \\
\hline External causes of injury and poisoning (V01-Y98) & $22(1.8)$ & $298(0.3)$ & 4.02 & 2.48 to 6.53 \\
\hline Accidental poisoning (X40-X49) & $4(0.3)$ & $18(0.0)$ & 13.64 & 3.98 to 46.73 \\
\hline Suicide and intentional self-harm (X60-X84) & $6(0.5)$ & $102(0.1)$ & 2.72 & 1.08 to 6.83 \\
\hline Events of undetermined intent (Y10-Y34) & $9(0.7)$ & $35(0.0)$ & 10.89 & 4.53 to 26.16 \\
\hline
\end{tabular}

\section{DISCUSSION}

This population-based study showed increased all-cause mortality among people who visited the ED and received an AUD diagnosis compared to those who did not receive an AUD diagnosis, while taking into consideration mental disorders other than AUDs, frequency of visits, year of entrance, age and gender.

The HR for all causes of death is almost double ( $H R=1.91)$, consistent with results from previous studies which have shown a significant relationship between alcohol abuse and increased mortality ratio. ${ }^{1-3} 16$ According to Roerecke and Rehm's ${ }^{2}$ meta-analysis, the relative risk for combined genders in population studies was 1.95 (CI 1.49 to 2.55). Care must be taken in this comparison since Roerecke and Rehm ${ }^{2}$ included studies which are either based on patients with a clinical diagnosis of AUD or on persons with AUD identified through surveys in the general population, while the present study is based on patients diagnosed at the ED.

In this study, the all-cause mortality was higher among men than women; however, the number of deaths in women was few and the increased mortality was not statistically significant. Considering the differences in genders, Roerecke and Rehm ${ }^{2}$ state that the RR for men was lower than that for women with AUD, and this was also consistent with previous meta-analysis. ${ }^{1}$ In an Icelandic study, included in the meta-analyses, ${ }^{2} 3$ Thorarinsson found a mortality ratio of 2.24 for alcoholic males ${ }^{17}$ and our HR of 1.88 (CI 1.44 to 2.47 ) for men does not contradict that finding since different methods were used in identifying the exposed groups.

Parlesak et $a l^{18}$ and Kinney ${ }^{19}$ suggested an explanation for the differences between genders-how women's bodies are more susceptible to alcohol. Our results indicated that men in the AUD group are more likely to die from most causes of death than men in the comparison group. Women in the AUD group are more likely than women in the comparison group to die from alcoholrelated diseases such as AUD, mental and behavioural disorders, diseases of the circulatory system and from events of undetermined intent. However, there were too few women in this study to allow us to generalise due to the small amount of data. Population-based studies on AUDs and mortality among women seem to be lacking, ${ }^{2}$ so this study has a unique position, however, small.

Alcohol is a major cause of chronic liver diseases worldwide ${ }^{20}$ and our results are certainly in agreement: the HRs for chronic liver disease and alcohol liver disease were 14.69 and 19.06 , respectively.

In this study, HR for AUD (ICD-10 code F10) is almost 50-fold. Unfortunately, this cause of death was not reported in recent meta-analysis. ${ }^{3}$ According to McDonald et $a l^{12}$ and Shultz et $a l^{21}$ AUDs are classified as alcoholrelated diagnosis with alcohol-attributable fraction 1 .

In this study, high ratios were found for suicide, accidental poisoning and events of undetermined intent. Previous studies have found an association between alcohol use and suicide. ${ }^{2} 6$ Mental disorders are also associated with suicide. ${ }^{6} 722$

Our findings show that patients in the AUD group are frequent users of the ED. A previous study from Stockholm, Sweden on AUD patients similarly showed that these patients were frequent users of ED. ${ }^{9}$ A recent study from Boston, Massachusetts showed that frequent users of the ED had alcohol-related visits and symptoms. ${ }^{10}$ A study from UK found that alcohol users are not only frequent users of medical services like EDs, but are also underusers of preventative services. ${ }^{23}$ Studies on unspecified frequent users of EDs indicate that they have poor physical and mental health, lack social support and are in socioeconomic distress. ${ }^{24} 25$ Frequent users of the ED have higher all-cause mortality than those in the general population ${ }^{26} 27$ and the risk for mortality from different causes increased with the 
Table 4 Number of all causes and selected causes of death among men and women separately among the AUD group and the comparison group HR, $95 \%$ Cls adjusted for age, number of visits, year of entrance and mental and behavioural disorders at discharge

\begin{tabular}{|c|c|c|c|c|}
\hline $\begin{array}{l}\text { Men } \\
\text { Causes of death (ICD-10) }\end{array}$ & $\begin{array}{l}\text { AUD group } \\
\text { p-yr } 3681 \\
\text { N (\%) }\end{array}$ & $\begin{array}{l}\text { Comparison group } \\
\text { p-yr } 216978 \\
\text { N (\%) }\end{array}$ & HR & $95 \% \mathrm{Cl}$ \\
\hline All causes (A00-Y98) & $57(7.4)$ & $2443(4.3)$ & 1.88 & 1.44 to 2.47 \\
\hline Mental and behavioural disorders (F00-F99) & $3(0.4)$ & $47(0.1)$ & 7.05 & 2.12 to 23.40 \\
\hline Alcohol use disorders (F10) & $3(0.4)$ & $5(0.0)$ & 41.27 & 8.23 to 206.91 \\
\hline Diseases of the circulatory system (100-199) & $20(2.6)$ & $863(1.5)$ & 2.09 & 1.33 to 3.28 \\
\hline Ischaemic heart diseases (120-125) & $2(0.3)$ & $202(0.4)$ & 1.12 & 0.28 to 4.54 \\
\hline Diseases of the respiratory system (J00-J99) & $3(0.4)$ & $139(0.2)$ & 1.79 & 0.55 to 5.82 \\
\hline Diseases of the digestive system (K00-K93) & $6(0.8)$ & $64(0.1)$ & 7.14 & 2.92 to 17.47 \\
\hline Chronic liver disease (K70, K73-K74) & $5(0.6)$ & $17(0.0)$ & 21.25 & 7.08 to 63.85 \\
\hline Alcoholic liver disease (K70) & $5(0.6)$ & $13(0.0)$ & 27.20 & 8.70 to 85.04 \\
\hline External causes of injury and poisoning (V01-Y98) & $20(2.6)$ & $190(0.3)$ & 5.10 & 3.03 to 8.60 \\
\hline Accidental poisoning (X40-X49) & $4(0.5)$ & $8(0.0)$ & 32.56 & 8.83 to 120.05 \\
\hline Suicide and intentional self-harm (X60-X84) & $6(0.8)$ & $74(0.1)$ & 3.90 & 1.53 to 9.96 \\
\hline Events of undetermined intent (Y10-Y34) & $7(0.9)$ & $16(0.0)$ & 14.13 & 4.76 to 41.90 \\
\hline Women & $\begin{array}{l}\text { p-yr } 2022 \\
\text { N (\%) }\end{array}$ & $\begin{array}{l}\text { p-yr } 185512 \\
\text { N (\%) }\end{array}$ & HR & $95 \% \mathrm{Cl}$ \\
\hline All causes $(A 00-Y 98)$ & $15(3.4)$ & $2364(4.8)$ & 1.63 & 0.98 to 2.71 \\
\hline Mental and behavioural disorders (F00-F99) & $1(0.2)$ & $80(0.2)$ & 8.42 & 1.14 to 62.00 \\
\hline Alcohol use disorders (F10) & $1(0.2)$ & $1(0.0)$ & 75.80 & 4.37 to 1317.01 \\
\hline Diseases of the circulatory system (100-199) & $8(1.8)$ & $783(1.6)$ & 4.00 & 1.98 to 8.06 \\
\hline Ischaemic heart diseases (I20-I25) & $1(0.2)$ & $164(0.3)$ & 2.48 & 0.34 to 17.81 \\
\hline Diseases of the respiratory system (J00-J99) & $1(0.2)$ & $195(0.4)$ & 2.01 & 0.28 to 14.47 \\
\hline Diseases of the digestive system (K00-K93) & $0(0.0)$ & $74(0.1)$ & - & - \\
\hline Chronic liver disease (K70, K73-K74) & $0(0.0)$ & $7(0.0)$ & - & - \\
\hline Alcoholic liver disease (K70) & $0(0.0)$ & $3(0.0)$ & - & - \\
\hline External causes of injury and poisoning (V01-Y98) & $2(0.5)$ & $108(0.2)$ & 1.57 & 0.37 to 6.72 \\
\hline Accidental poisoning $(\mathrm{X} 40-\mathrm{X} 49)$ & $0(0.0)$ & $10(0.0)$ & - & - \\
\hline Suicide and intentional self-harm (X60-X84) & $0(0.0)$ & $28(0.1)$ & - & - \\
\hline Events of undetermined intent (Y10-Y34) & $2(0.5)$ & $19(0.0)$ & 6.77 & 1.32 to 34.82 \\
\hline
\end{tabular}

increasing number of visits to the $\mathrm{ED}$, in a doseresponse manner. ${ }^{27}$ The increased mortality was not only due to natural causes of death, but also due to external causes, such as accidental poisoning and suicide, ${ }^{26} 27$ which may indicate help-seeking behaviour that was unmet. These issues are, besides the statistical aspects, a further justification for including the number of visits as a covariate in this study.

Concern may arise as to whether AUD cases are seeking wards at the psychiatric ED, general practitioners or other healthcare services and therefore underrepresented at the general ED in this study. If this is the case, it would eventually lead to a smaller sample of AUD patients at the ED; however, this will not disturb the classification of the patients in the study groups or necessarily bias the comparison between the groups in the study. Under-representativeness of AUD cases at the ED is unlikely as the generally held view that heavy alcohol consumers are frequent users of medical services and the accident and EDs is supported by a detailed study. ${ }^{23}$

\section{Strength}

The use of comprehensive population registries and the personal identification number, which enabled easy and accurate record linkage, strengthens the study. Thus, vital status was ascertained through the National Cause-of-Death Registry for all individuals, in the same way for the AUD group and the comparison group. Only $0.2 \%$ of the causes of death among the total cohort were reported on the death certificates as due to unknown and unspecified causes, indicating the quality of the information on the death certificates. The autopsy rate is about $14 \%$ of all deaths during the study period. ${ }^{14}$

Death certificates in Iceland are issued by a physician. If the deceased person's physician is not able to attest the cause of death, or in cases where the circumstances of the death are unexplained, unusual, suspicious, due to intoxication or following an accident, the death is reported to the police and the medical examiner, who arrange for an autopsy and forensic investigations before the death certificate is issued. ${ }^{28}$ Mather $e t a l^{29}$ studied death registration at a global level; the quality of 
registration data from Iceland was categorised as high overall and ranked in the same category as data from 23 developed countries, including the USA and the UK.

The universal use of the personal identification number in the files of the ED enabled an accurate registration of whether and when the patients made repeated visits to the ED through record linkage, as well as counting the number of visits and identifying the discharge diagnosis. The setting is favourable for counting the number of visits to the ED and the discharge diagnosis, since the ED and the hospital were the only acute healthcare institutes of this kind serving the population in the catchment area and therefore did not face any competition from other similar institutes.

\section{Limitations}

The study material originates from a single academic healthcare institution in an urban setting, serving as the number one trauma centre and community hospital. This may limit the generalisability of the results. However, the size and the characteristics of the background population are known and the population is homogeneous, being 99\% white Caucasian, with uniform financing within the healthcare system. In this prospective cohort study, we used the cumulative clinical information at the ED, which may lack systematisation but nevertheless is considered to reflect the routine operations of the ED, and the comprehensive population registries in a prospective design.

Another limitation of this study is the sole use of the main diagnosis at discharge from the $\mathrm{ED}$, and we have only taken these diagnosis into account as an ever/never phenomenon. Many of the users of the ED surely also had other diagnoses than the main diagnosis, reported in the paper records not registered in the computerised records. Our procedure using only the main diagnosis does not ensure that we have identified all patients with mental disorders, and so there is a possibility of residual confounding due to mental disorders. Nevertheless, the main diagnosis at discharge is considered to reflect the main clinical evaluation of the attending physician, taking into account the patient's symptoms and his/her condition at the time of the visit. ${ }^{30}$

There is an inherent weakness in cohort studies comparing the mortality of severe cases of AUD with that of the general population, since the majority of those with AUD in the general population are undiagnosed and never receive treatment, as pointed out in the earlier discussion. ${ }^{2}$ The comparison group in these studies is contaminated with a considerable number of alcoholics, thus underestimating the mortality risk. Similar previous comparative studies, which are based on population surveys for AUDs, may also be handicapped due to a lack of accuracy in the diagnostic process (not clinical) and the fact that some of those with alcohol dependency or misuse are in denial, and are not detected in the surveys, but remain in the comparison groups.
In accordance with this discussion, the increased mortality risk found in this study may also be an underestimation.

In a sensitivity analysis we excluded patients with selected alcohol-related main diagnosis, such as alcohol poisoning (ICD-10 code T51), or alcohol liver disease (ICD-10 code K70), from the groups, and the HR did not change substantially, so the main results are not seriously underestimated for this reason.

The patients in this study were confined to those discharged home from the ED after diagnostic workup and initial treatment. Patients visiting the ED, who were admitted to a hospital ward, may differ from those who were discharged home. One may postulate that when patients were admitted to the hospital ward they were considered to be seriously ill or with severe injuries, and they were definitely under observation and treatment for a day or more, while those treated only at the ED did not stay there for more than a few hours. The physicians of the $\mathrm{ED}$ are responsible for the diagnosis and treatment of the discharged patients. Thus, the generalisability of this study may be limited to patients discharged from the ED.

\section{CONCLUSION}

AUD as the discharge diagnosis at the ED, among patients who were not admitted to a hospital ward but discharged home, predicts high overall mortality when taking into consideration age, gender, mental disorders, year of entrance and number of visits. Mental disorders from previous studies are known to be related to increased mortality, in this study reconfirmed, while number of visits to the ED may be a new risk indicator among those with AUD. The mortality was specifically increased for alcohol-related diseases such as mental and behavioural disorders due to alcohol, and alcohol liver diseases, as well as for diseases of the circulatory system, accidental poisoning, suicide and events of undetermined intent. As the results conclusively show the vulnerability of these patients, one can question whether their needs are adequately met at the ED.

Contributors ASG, AK, RG, OSG and VR substantially contributed to the conception and design, obtained the data and analysed and interpreted the data; drafted the article and revised it critically for important intellectual content; and approved the final version of the submitted manuscript.

Funding This study was supported by grants from Landspitali-the National University Hospital Research Fund and the Icelandic Nurse's Association Research Fund, grant number 311055-2249; and the University of Iceland Research Fund, grant number 1238-123368.

Competing interests None.

Ethics approval The National Bioethics Committee (VSNb2009020009/03.7), the Ethical Committee of the Landspitali University Hospital, and the Data Protection Commission (2009020152BRA/-) approved the study.

Provenance and peer review Not commissioned; externally peer reviewed.

Data sharing statement No additional data are available.

Open Access This is an Open Access article distributed in accordance with the Creative Commons Attribution Non Commercial (CC BY-NC 4.0) license, which permits others to distribute, remix, adapt, build upon this work noncommercially, and license their derivative works on different terms, provided the original work is properly cited and the use is non-commercial. See: http:// creativecommons.org/licenses/by-nc/4.0/ 


\section{REFERENCES}

1. Harris EC, Barraclough B. Excess mortality of mental disorder. Br J Psychitry 1998;173:11-53.

2. Roerecke M, Rehm J. Alcohol use disorders and mortality: a systematic review and meta-analysis. Addiction 2013;108:1562-78.

3. Roerecke M, Rehm J. Cause-specific mortality risk in alcohol use disorder treatment patients: a systematic review and meta-analysis. Int J Epidemiol 2014;43:906-19.

4. John U, Rumpf HJ, Bischof G, et al. Excess mortality of alcohol-dependent individuals after 14 years and mortality predictors based on treatment participation and severity of alcohol dependence. Alcohol Clin Exp Res 2013;37:156-3.

5. Markkula N, Harkanen T, Perala J, et al. Mortality in people with depressive, anxiety and alcohol use disorders in Finland. Br J Psychiatry 2012;200:143-9.

6. Harris EC, Barraclough B. Suicide as an outcome for mental disorders. A meta-analysis. Br J Psychitry 1997;170:205-28.

7. Flensborg-Madsen T, Knop J, Mortensen EL, et al. Alcohol use disorders increase the risk of completed suicide-irrespective of other psychiatric disorders. A longitudinal cohort study. Psychiart Res 2009;167:123-30.

8. Hasin DS, Stinson FS, Ogburn E, et al. Prevalence, correlates, disability, and comorbidity of DSM-IV alcohol abuse and dependence in the United States: results from the National Epidemiologic Survey on Alcohol and Related Conditions. Arch Gen Psychiatry 2007;64:830-42.

9. Hansagi $\mathrm{H}$, Engdahl B, Romelsjo A. Predictors of repeated emergency department visits among persons treated for addiction. Eur Addict Res 2012;18:47-53.

10. Liu SW, Nagurney JT, Chang Y, et al. Frequent ED users: are most visits for mental health, alcohol, and drug-related complaints? Am J Emerg Med 2013;31:1512-15.

11. Malone D, Friedman T. Drunken patients in the general hospital: their care and management. Postgrad Med J 2005;81:161-6.

12. McDonald AJ III, Wang N, Camargo CA Jr. US emergency department visits for alcohol-related diseases and injuries between 1992 and 2000. Arch Intern Med 2004;164:531-7.

13. Gunnarsdottir OS, Rafnsson V. Non-causative discharge diagnosis from the emergency department and risk of suicide. J Emerg Med 2010;38:286-92.

14. Statistics I. Statistics 2013 [cited 2013 02.12.2013]. http:www.statice is/Pages/422

15. Cox DR. Regression models and life-tables. J Royal Statist Soc Series B (Methodological) 1972;34:187-220.
16. Britton A, McKee M. The relation between alcohol and cardiovascular disease in Eastern Europe: explaining the paradox. $J$ Epidemiol Communit Health 2000;54:328-32.

17. Thorarinsson AA. Mortality among men alcoholics in Iceland, 1951-74. J Stud Alcohol 1979;40:704-18.

18. Parlesak A, Billinger $\mathrm{MH}$, Bode $\mathrm{C}$, et al. Gastric alcohol dehydrogenase activity in man: influence of gender, age, alcohol consumption and smoking in a caucasian population. Alcohol Alcohol 2002;37:388-93.

19. Kinney J. Loosening the grip. A handbook of alcohol information. 8th edn. New York: McGraw-Hill Company, 2006.

20. Rehm J, Taylor B, Mohapatra S, et al. Alcohol as a risk factor for liver cirrhosis: a systematic review and meta-analysis. Drug Alcohol Rev 2010;29:437-45.

21. Shultz JM, Rice DP, Parker DL, et al. Quantifying the disease impact of alcohol with ARDI software. Public Health Rep 1991;106: 443-50.

22. Pompili M, Serafini $\mathrm{G}$, Innamorati $\mathrm{M}$, et al. Suicidal behavior and alcohol abuse. Int J Environ Res Public Health 2010;7: 1392-431.

23. Cryer PC, Jenkins LM, Cook AC, et al. The use of acute and preventative medical services by a general population: relationship to alcohol consumption. Addiction 1999;94 1523-32.

24. Olsson M, Hansagi $\mathrm{H}$. Repeated use of the emergency department. Qualitative study on the patient's perspectives. Emerg Med J 2001;18:430-4

25. Mandelberg JH, Kuhn RE, Kohn MA. Epidemiological analysis of an urban, public emergency depatment's frequent users. Acad Emerg Med 2000;7:637-46.

26. Hansagi $\mathrm{H}$, Allebeck $\mathrm{P}$, Edhag $\mathrm{O}$, et al. Frequency of emergency department attendances as a predictor of mortality: nine years follow-up of a population based cohort. J Public Health Med 1990;12:39-44.

27. Gunnarsdottir OS, Rafnsson V. Mortality of users of a hospital emergency department. Emerg Med J 2006;23:269-73.

28. Log um danarvottord, krufningar og fleira. 1998 [cited 28 Nov 2013] 61. http://althingi.is/lagas/nuna/1998061.html

29. Mathers CD, Fat DM, Inoue M, et al. Counting the dead and what they died from: an assessment of the global status of cause of death data. Bull World Health Organ 2005;83:171-7.

30. Rafnsson V, Gunnarsdottir OS. All-cause mortality and suicide within 8 days after emergency department dischage. Scand J Public Health 2013;41:832-8. 